\title{
La Polémica en torno a los manicomios, 1916.
}

\author{
Olga Villasante. \\ Psiquiatra. Unidad de Psiquiatría, Hospital Severo Ochoa. Leganés (Madrid). \\ ovillasante.hsvo@salud.madrid.org
}

El surgimiento de la llamada "primera psiquiatría científica" en las primeras décadas del siglo XX en España, está asociado a la generación de Archivos de Neurobiología $(1,2)$. Un grupo de hombres de la talla de José Miguel Sacristán (1887-1957), Enrique Fernández Sanz (1872-1950) o César Juarros (1879-1942), por citar sólo algunos de ellos, participaron en un proceso de renovación política, cultural y científica que les llevó a formarse en psiquiatría y neurología en el extranjero, muchas veces ayudados por las becas que la Junta de Ampliación de Estudios (3). Sus visitas a clínicas francesas y alemanas generaron inquietudes en esos médicos y científicos españoles con una consiguiente actitud crítica hacia los escasos establecimientos psiquiátricos existentes y la atención dispensada al enfermo mental.

El artículo de Gonzalo Rodríguez Lafora (1886-1971) publicado el 12 de octubre de 1916 en España, bajo el título "Los manicomios españoles" (4), se puede inscribir en este contexto y ha sido citado, en múltiples ocasiones, como el ejemplo de las precarias condiciones en que se hallaban los enfermos psiquiátricos en las instituciones españolas. Lo novedoso y, de algún modo, "escandaloso" de este artículo de denuncia fue, sin duda, la reproducción de fotografías de enfermos psiquiátricos en las que se mostraban pacientes ingresados en el Manicomio Provincial de Granada y el Manicomio Provincial de Valencia. Si bien el análisis de estas imágenes de enfermos en lechos de paja, desnudos o cubiertos únicamente con una manta y de pacientes que presentaban los pies y las manos encadenadas a un cinturón de hierro, ya fue motivo de una entrada en el interesante blog $^{1}$ que Oscar Martínez Azumendi ha enriquecido en los tres últimos años, se trata, en esta ocasión, de escrutar algunos de los contenidos del texto que acompañó a las inquietantes fotografías.

El objetivo de esta segunda entrega de "Historias con Historia" es, pues, la reproducción una serie de cuatro artículos de prensa que empiezan con el ya citado de Lafora -apellido por el que ha sido citado habitualmente este psiquiatra valenciano-. A ésta dura crítica (4) le siguió una rápida respuesta de Ricardo Pérez Valdés (5), una contrarréplica (6) y, en el número de la semana siguiente a esta, una intervención de Tomás Busquet i Teixidor (7). Se describirán algunas pinceladas sobre esta polémica periodística y sus protagonistas, si bien la lectura de los textos que se reproducen a continuación, habla por

${ }^{1}$ http://www.psiquifotos.com/2009/10/89-los-manicomios-espanoles-de-lafora.html

Trabajo realizado en el marco del Proyecto de Investigación financiado por el Ministerio de Ciencia e Innovación, HAR08-04899-C02-01. 
sí sola. Comentaremos, además, a modo de epílogo, un documento titulado Lo que debe ser un manicomio Provincial. Apuntes recopilados por Ignacio Bauer (8), menos conocido y que, probablemente, fue también consecuencia de la intervención de Lafora. Si bien éste no vio la luz hasta 1925, publicado por el entonces Visitador de Manicomios y Presidente de la Comisión de Beneficencia de la Excma Diputación Provincial de Madrid, incluía un informe de R. Pérez Valdés firmado el 2 de abril de 1917, es decir sólo unos meses después del artículo de Lafora.

Las deficiencias de las instituciones psiquiátricas en las primeras décadas del siglo XX

Si la fotografía como medio de denuncia social adquiere su máximo potencial a mediados del siglo $\mathrm{XX}$, al convertirse los fotógrafos en testigos y vehiculizadores de las carencias y defectos de las instituciones psiquiátricas, instando a una transformación de la psiquiatría (9), las instantáneas aportadas por Lafora se pueden considerar un precedente en nuestro país. Estas imágenes aparecieron, como ya hemos dicho, en Espa$\tilde{n} a$, revista subtitulada Semanario de la Vida Nacional, que según se recoge en la Hemeroteca Nacional, fue la publicación de más amplia repercusión en la denominada "Edad de Plata" de la intelectualidad española. Este primer proyecto periodístico de José Ortega y Gasset (1883-1955), en el que confluyeron las corrientes reformistas liberal-democrática, las antioligárquicas, radicales y antimonárquicas del socialismo, se dieron cita tanto los máximos representantes de la generación del 98 como la del 14, y algunos que integrarán la del 27 y nació de un espíritu neo - regeneracionista en torno a la Liga de Educación Política. Así pues, resultó impactante que una revista de difusión general en la que participaron ilustres como Pío Baroja, Pérez de Ayala o Azorín, recogiera esta crítica a las instituciones españolas, ya proclamada por profesores extranjeros, unos años antes, en el Congreso Internacional de Medicina de Madrid, en 1903.

Si bien el objetivo de Lafora no parece un ataque específico al departamento de alienados del Hospital Provincial de Madrid, al que dedicó poco más de una docena de líneas de su artículo, fue precisamente este párrafo el origen de la polémica periodística que nos ocupa. Además de las ya citadas fotografías de los enfermos de las instituciones granadina y valenciana, describió una trágica situación en el departamento de alienados del Hospital Provincial que, como luego veremos, replicó inmediatamente R. PérezValdés (1853-1927).

De hecho Lafora, en este artículo realizó una introducción en la que ejemplifica el atraso de la sociedad española con las instituciones manicomiales que, en general, considera insalubres, sucias y abandonadas. No excluye de culpabilidad a los psiquiatras a quien acusa de "ignorantes o abandonados, no han levantado nunca una voz de protesta que llegase hasta nuestros gobernantes" (4, p.8). Después de citar autores europeos como Janet, Freud o Kraepelin, relata que ha visitado los manicomios provinciales de Cádiz, Toledo, Granada, Valencia y Madrid, si bien a los dos primeros no les dedica ninguna crítica en particular. Realizó especial hincapié en las condiciones arquitectónicas de las instituciones fotografiadas, así como en la utilización de métodos coercitivos ya desechados como cadenas o esposas. Criticaba estos métodos aludiendo al humanitarismo y al cientifismo que había llevado a enunciar el non-restraint y señalaba la falta de departamentos balneoterápicos. 
Los "guardianes" de los pacientes no recibieron muchos halagos de Rodríguez Lafora y, al referirse a los vigilantes de Valencia los describe como "hombres ineducados, sucios, desgarbados y de una jovialidad amable". Por otra parte en su visita al Manicomio de Granada, un bello edificio del Renacimiento, describió que "un ceñudo guardián con recio manojo de enormes llaves nos va abriendo las sólidas puertas de estas celdascalabozos" (4, p.8). Destacó como defectos comunes al resto de los establecimientos, la insuficiencia de personal, tanto médico como de enfermería, así como su escasa profesionalización. La escasa presencia médica en los establecimientos ya percibida por E. C. Seguin (1843-1898) en su visita durante el invierno 1882-1883 a múltiples instituciones psiquiátricas, fue reflejada durante el Certámen Frenopático Español (10). Es de sobra conocido que la psiquiatría, en la segunda década del siglo XX, aún no estaba reconocida como especialidad y que si algún médico se interesaba por el estudio de las enfermedades nerviosas o mentales, debía aprender junto a algún colega, podía asistir a algunas lecciones que se impartieron en el Hospital Provincial o, como hemos referido, viajar a clínicas europeas.

Además de las escasas posibilidades para los médicos de formarse en patología mental, también se estaba reclamando la profesionalización de los enfermeros y, en las siguientes décadas, coincidiendo con la Segunda República, podemos detectar los primeros pasos para la creación de la especialidad que, como sabemos, aún ha tardado casi un siglo en consolidarse de modo oficial en la enfermería de salud mental. De hecho, a partir de la Órden ministerial del 16 de mayo de 1932, en la que se reconoció el Diploma de "enfermero psiquiátrico", se iniciaron actividades formativas como los cursillos para la preparación teórica y práctica en el Manicomio
Provincial de Valencia, cuyos contenidos se materializaron en el Prontuario del enfermero psiquiátrico publicado por Domingo Simó en 1936 (11). También en Madrid, el 18 de septiembre de 1933 se comenzó un curso para practicantes, enfermeros y enfermeras psiquiátricos, cuya parte teórica fue impartida, por las tardes en el "Hospital de la Beneficencia General" por Mendiguchía Carriche, Fernández Méndez, Martín Vegué y Moreno Rubio, todos ellos médicos del manicomio de Leganés (12), y la parte práctica en esta última institución en días festivos -Fernández Sanz, jefe facultativo entonces del Manicomio Nacional, pronunció la conferencia de clausura (13)-. Sin embargo, a pesar de estas y otras iniciativas sobre la formación de enfermería psiquiátrica en los años treinta, la realidad es que, en la segunda década del siglo, todavía los enfermeros, cuidadores, mozos o guardianes accedían a las instituciones sin una formación específica.

El departamento de alienados del Hospital Provincial de Madrid

"Del departamento de alienados del Hospital Provincial de Madrid, sólo diremos que permanece en el mismo estado que en la época de su fundación (1748). Allí no existen baños para los agitados, a los que sujeta mediante camisas de fuerza, otro utensilio desterrado ya de todas partes. Cuando se empiezan a acumular los enfermos una monja ordena traslados en masa a otros manicomios (al de Ciempozuelos o al de Valladolid), y los enfermos llegan a estos últimos con el único diagnóstico de enagenación mental y sin ningún dato ulterior que sirva para diagnosticarlos científicamente. Aún reciente es la publicación de agresiones de sus guardianes a un alienado" $(4$, p. 9$)$. 
Estas líneas de Lafora son las que ocasionaron la réplica de Ricardo Pérez Valdés y Aguirre que, primeramente, acusa al psiquiatra valenciano de desprestigiar todos los manicomios españoles a partir de algunos casos, defendiendo que otros manicomios en Cataluña, Navarra, Zaragoza, Santiago, Valladolid... si bien no eran modelo de asistencia "podían rivalizar ventajosamente con los el Sr. Lafora haya visto en Inglaterra, Francia o Alemania" (5). Ricardo Pérez Valdés, que era natural de Santander y se había trasladado a Madrid después de finalizar el bachillerato en 1869 para licenciarse en Medicina en 1873, había estado a cargo del servicio de dementes durante los ocho años previos a esta polémica. Se había formado en Paris, entre 1878 y 1879 con profesores como Charcot $\mathrm{y}$, en el año 1881 se había presentado a la oposición de 6 plazas de médico de la Beneficencia Provincial, de modo que desempeñó, primero como médico de guardia en el hospital madrileño y, simultáneamente, a petición propia, encargado, con carácter interino, de las salas que visitaba el prestigioso José $\mathrm{M}^{\mathrm{a}}$ Esquerdo y Zaragoza (1842-1912), hasta que, en 1885, le correspondió ascender a médico de sala (14).

Así pues, el 18 de octubre de 1916 en La Correspondencia de España (5), Pérez Valdés calificaba las declaraciones de su colega de alarmistas y estremecedoras y trataba de justificar y defender el departamento que gobernaba. Es verdad que las salas de enajenados del Hospital Provincial, denominadas de S. Isidro y Santa María de la Cabeza (números 55 y 46) habían sido descritas, a mediados del siglo XIX (15), como lóbregas, inmundas y miserables por José Rodríguez Villargoitia (1811-1854) (16), quien había realizado, en 1846, un proyecto para la construcción de un nuevo Departamento. Esta reforma no se consolidó y, en la Memoria del año 1874, se describe que las salas estaban en los sótanos del hospital, carentes de condiciones higiénicas, sin luz, ni agua, ni ventilación y que difícilmente se ajustaban las condiciones exigidas por los principios terapéuticos de la especialidad. Se había convertido en un "depósito subterráneo", en el que sólo algunos pacientes podían ser trasladados al Hospital Provincial de los Inocentes (el Nuncio) de Toledo, San Baudilio de Llobregat o al Manicomio de Leganés (17).

Sin embargo, Pérez Valdés afirmó que las salas decimonónicas se habían cambiado por "salas amplias, llenas de luz y de aire, pavimentadas con lujo... y amplios patios". No es fácil objetivar las afirmaciones tan encontradas de ambos polemistas, ya que no abunda la bibliografía sobre este departamento de observación de dementes, dónde según el Decreto del 1885, vigente hasta la implantación del Decreto de julio de 1931, los pacientes debían permanecer hasta que se les condujera a un manicomio en clase de "reclusos permanentes" (18). Durante la última década del siglo trabajaron allí José $\mathrm{M}^{\mathrm{a}}$ Esquerdo y Jaime Vera y López (1858-1918), vinculado al nacimiento del socialismo en España y definido como el primer teórico del marxismo español, quien había ocupado una plaza por oposición en el Hospital General desde 1884. En una carta autógrafa de 1891 de Esquerdo, el célebre psiquiatra que aparecía como "titular de la Sala de Observación de Enajenados", planteaba la dificultad para trasladar a los pacientes cuyos expedientes "dementes reclusos" estaban terminados y se quejaba, asimismo, del hacinamiento de enfermos, ya que se encontraban 87 enfermos en un espacio cuya capacidad era para 30 o 40 plazas $(19,20)$.

Desconocemos si la situación del departamento había mejorado respecto a la decimonónica, pero no es la percepción que tuvo Lafora a juzgar por las declaraciones vertidas en España. Al contrario, el director 
del Departamento de Dementes defendió que la Hermana de La Caridad que prestaba sus servicios en el departamento era eficiente, abnegada y no traspasaba los límites de sus funciones interviniendo en los traslados. Sin embargo no era la primera vez que los médicos denunciaban la intervención excesiva de las religiosas en otras instituciones psiquiátricas (21), en parte por ausencia de los médicos que, muchas veces trabajaban simultáneamente en diferentes establecimientos y consultas. El mismo Pérez Valdés, mientras era médico de esta sala de enfermedades mentales mantuvo una intensa actividad que le llevó a dirigir el lazareto que se instaló en el cerro de Los Ángeles para el control de la epidemia de cólera de 1885, colaboró en la Revista Clínica de Hospitales y la Revista de Especialidades, fue consejero de Sanidad y atendió una numerosa consulta de clientela privada.

La anotación de registros clínicos era de obligado cumplimiento en otras instituciones como la de Leganés, cuestión que se hallaba recogida en el Reglamento interno de la institución (22), sin embargo el director del departamento del Hospital Provincial se disculpaba de esta obligación alegando que seguía con "la costumbre establecida por los ilustres frenópatas que me precedieron". Es posible que el tono crítico de Rodríguez Lafora fuese un tanto alarmista y probablemente influido por su formación en Berlín y Munich y su estancia como patólogo en el Government Hospital for Insane de Washington. Había regresado a España en 1912 $\mathrm{y}$, durante esa década se dedicó fundamentalmente a labores de investigación, entre las que se destaca el laboratorio de Fisiología Cerebral anejo a la Residencia de Estudiantes y tareas derivadas de su actividad en el Patronato Nacional de Subnormales (23), pero no había trabajado en ningún establecimiento psiquiátrico (24). Su vehemente criti- ca de la institución madrileña, si bien no atacaba directamente a los médicos, no es raro que ocasionara la reacción del Pérez Valdés que trató de defender, no se si de modo suficientemente convincente, las condiciones del departamento. El médico santanderino describió mejoría en las condiciones de alojamiento como las camas con colchón que habían sustituido a los flacos jergones de paja o la cocina especial dentro del departamento y justificó la utilización de la camisa de fuerza, definida como aquella "blusa de lona, cuyas mangas, más prolongadas que de ordinario, terminan en sendas cintas que, cruzándose en la parte anterior del cuerpo vienen a atarse en la espalda, impidiendo los movimientos demasiado amplios de los brazos é inutilizando el uso de las manos" (5). Si bien Lafora refería que este método de sujeción había sido abandonado o desterrado, es, a mi modo de entender, una aseveración propia de alguien alejado de la práctica asistencial y, quizá, un tanto demagógica. Parece difícil de pensar que, hace casi un siglo, en la era prefarmacológica se pudiese tratar a pacientes con riesgo elevado de auto y heteroagresión sin ningún método de contención. Un argumento similar utilizó Pérez Valdés, en la réplica, al relatar que un amigo particular de Lafora que había ejercido como interino en el departamento de enajenados -quizá podría referirse a Achúcarro o Sanchís Banús- había intentado su supresión hasta que recibió una agresión.

Lafora inició con esta denuncia una serie de polémicas periodísticas que caracterizaron su trayectoria profesional, ya que, poco después, en 1917, se enfrentaba a Burell, Ministro de Instrucción Pública acusándole de favoritismos y arbitrariedades en el funcionamiento del Patronato de anormales. Después polemizó con la epidemia de gripe de 1918 en el Ejército, el asesinato de Hildegart, criticó la Facultad de Medicina y en- 
tabló una conocida polémica por la vacante de Santiago Ramón y Cajal (1852-1934), en la Academia Nacional de Medicina (3, pp. 99-127). Curiosamente, su crítica a las salas del Provincial no le impidió presentarse a la oposición convocada tras el fallecimiento repentino del valenciano José Sanchís Banús (1890-1932) el 22 de julio de 1932. Esta vacante iba a generar una enconada disputa al enfrentarse en la oposición con José M. Villaverde y Larrar (1888-1936), que derivó en la división del servicio de dementes, de modo que Lafora se encargó de la sección de mujeres y Villaverde de los hombres (25, 26).

Lafora finalizaba el primer artículo instando a las diputaciones a cumplir "sus obligaciones benéficas", para lo que se precisaban grandes cantidades de dinero y, en la contrarréplica, descargaba de la responsabilidad de la organización del anticuado departamento a Pérez Valdés reconociendo su valía profesional (6). Sin embargo, nuevamente utilizaba argumentos comparativos con instituciones como el Psychiatric Ward de Nueva York y aún redundaba más en la escasa presencia médica, la inexistencia de un médico especialista residente, la escasez de personal de enfermería suficientemente cualificado, cuestiones, en cualquier caso, que califica como comunes en la mayor parte de las instituciones españolas.

Y para finalizar la polémica que nos ocupa, el médico inspector del Servicio de dementes de la Mancomunitat de Cataluña, Tomás Busquet, intervino para apoyar la crítica de Lafora, en el número 93 del Semanario España (7). Este psiquiatra, cuya principal producción estuvo relacionada con la organización de los servicios psiquiátricos y con la divulgación de la Higiene mental que plasmó en La lucha con los trastornos del espíritu. Higiene Mental Popular (27), también consideraba que los servicios de demen- tes en los hospitales públicos eran nefastos. Los cambios más significativos a realizar no estaban sólo en los viejos edificios, sino en el sistema de organización en el que prácticamente no se podía implantar el open-door y el no-restraint. También culpabiliza a los médicos de dicha situación a quienes considera responsables del ambiente de coerción y represión presente en los establecimientos.

Por otra parte alabó el plan de reorganización de la Mancomunidad catalana y explicaba que disponía de tres niveles de asistencia: un hospital Mental para las psicosis agudas, asilos-colonias para los alienados crónicos y asilos especiales para los atrasados, alienados difíciles, epilépticos. La Mancomunitat había adquirido una extensión de más de 30 hectáreas para un hospital mental en el que, según el inspector catalán, se modificarían los principios terapéuticos, se practicaría "encamamiento, psicoterapia y baños" y se suprimiría la coerción mecánica. Cataluña, de hecho, poseía ya desde el siglo XIX, una red asistencial significativamente más amplia que la madrileña y algunas estructuras que impulsaron la transformación de la asistencia psiquiátrica. En 1911, se había fundado la Societat de Neurologia $i$ Psiquiatria, presidida por Arturo Galcerán Granés (1850-1919), fundamental en el proceso de reforma de la red asistencial catalana (28).

A modo de epílogo: La respuesta de la Comisión de Beneficencia de la Diputación Provincial de Madrid

Intuimos que la crítica de Lafora realizada en octubre de 1916 pudo determinar que, en 1917, el Decano de la Beneficencia Provincial de Madrid solicitara un informe a Ricardo Pérez Valdés sobre Lo que debe ser 
un manicomio Provincial (8). El texto escrito por el responsable del Provincial, firmado el 2 de abril de 1917, no se publicó hasta 1925, "por razones que prefiero ignorar", en palabras de Ignacio Bauer, entonces Visitador de Manicomios y Presidente de la Comisión de Beneficencia de la Excma Diputación Provincial de Madrid-. La Memoria de Pérez Valdés, destinada a describir la distribución y organización de un Manicomio que proyectaba la Diputación de Madrid, ocupaba 24 páginas de los Apuntes recopilados por el citado Ignacio Bauer y Landauer (1891-?), que llegó a ser Presidente interino de la Diputación Provincial y correspondiente de la Real Academia de la Historia. Lo que debe ser un manicomio Provincial incluyó, además, un informe en el que resumía la situación de los locos y los manicomios a lo largo de la historia para describir, además, la legislación que regulaba los internamientos en España (8). Los apuntes recogieron, al final de la publicación, dos cartas dirigidas a Brauer; la primera de Ricardo Pérez Valdés y la segunda de Binswanger, ambas escritas en noviembre de 1924 y dos apéndices: "De interés social. En la inclusa" y "Una campaña Mundial. Hay que impedir que los seres degenerados, los locos, los enfermos y los alcohólicos engendren hijos". Este último apéndice recogía la opinión del Parlamento de Sajonia proponiendo la esterilización quirúrgica de multitud de enfermos (demencia precoz, locura maníaco-depresiva, epilepsia, degeneración alcohólica...), acorde a las orientaciones de una campaña sanitaria mundial. Si bien la discusión del contenido de este breve opúsculo implicaría una amplia discusión en relación con el discurso eugenésico que se aparta del tema que nos ocupa.

La carta de Pérez Valdés relata que nadie había reparado en su memoria hasta entonces, ya que, a pesar de ser encargada en 1917, sólo recibió notificación de ella siete años más tarde. Por otra parte, la de Binswanger alabó las notas de su colega, pero realizó algunas sugerencias bibliográficas como Bleuler y Kraepelin e insistió en la necesidad de instaurar la enseñanza de psiquiatría en la Universidad.

Así pues, el informe de Pérez Valdés para un proyectado manicomio en Madrid ni era el primero, ya que diversos proyectos se habían sucedido en las décadas previas, ni sería el último, como bien se ilustró en el original "De visita al manicomio" que abrió esta sección en el número previo de la revista de la AEN (29). Si bien es verdad que, durante el primer tercio de siglo, se multiplicaron los informes, debates y discusiones, en diferentes ámbitos, como la intervención de Fernández Sanz, en 1921, en la que disertó sobre la necesitada reforma de las instituciones (30), éstas no fueron suficientes para una transformación íntegra de la asistencia. Posiblemente el artículo de Lafora y la polémica desatada por él contribuyó a algunas mejoras, al menos, en el Hospital Provincial como la inauguración de un pabellón psiquiátrico de 77 camas contiguo al Hospital Provincial en febrero de 1932. Sin embargo, el planteamiento de una amplia reforma de las instituciones psiquiátricas españolas topó con una guerra que aún iba a empeorar más las condiciones de los enfermos psiquiátricos. Más de 50 años después del artículo de Lafora, el Manicomio Provincial de Valencia volvía a ser protagonista maldito de un reportaje en Sábado Gráfico (31), en la que aparecían las terribles condiciones de los enfermos mentales, también reproducidas en la entrada 34 del blog antes aludido.

\section{BIBLIOGRAFÍA:}

(1) Lázaro, J. Archivos de Neurobiología: los setenta y cinco años de la psiquiatría española. Archivos de Neurobiología 1995;58(1):13-30. 
(2) Campos, R., Huertas, R., Estado y asistencia psiquiátrica en España durante el primer tercio del siglo XX. Rev. Asoc. Esp. Neuropsiq 1998;18(65):99-108.

(3) Valenciano, L. El Dr. Lafora y su época. Madrid: Morata, 1977.

(4) Rodríguez Lafora, G. Los manicomios españoles. España. 12 oct 1916. 90:8-10.

(5) Pérez Valdés, R. Los manicomios Españoles. La Correspondencia de España. 18 oct. 1916 (2a ed.) 21434:4.

(6) Rodríguez Lafora, G. El manicomio provincial de Madrid (Réplica al Dr. Pérez Valdés). España. 26 oct. 1916. 92:8.

(7) Busquet, T. Manicomios españoles (Carta abierta al Dr. Gonzalo R. Lafora). España. 2 nov. 1916. 93:14.

(8) Bauer I. Lo que debe ser un manicomio Provincial. Apuntes recopilados por Ignacio Bauer. Madrid: Imprenta de Jesús López, 1925

(9) Martínez Azumendi, O. Periodistas y reporteros gráficos como agentes de cambio en psiquiatría. Rev. Asoc. Esp. Neuropiq. 2005;15(96):9-28.

(10) Villasante, O. Primer Certámen Frenopático Español (1883): estructura asistencial y aspectos administrativos. Asclepio1997;49(1):79-94.

(11) Simó, D. Prontuario del enfermero psiquiátrico Valencia: Imp. V. Climent Vila, 1936.

(12) Tierno, R., Villasante, O., Vazquez de la Torre, P. El manicomio Nacional de Leganés: entre la reforma legislativa y la práctica asistencial (1931-1936). En Campos, R., Villasante, O., Huertas, R. (Eds.).De la Edad de Plata al exilio. Construcción y reconstrucción de la psiquiatría española. Madrid: Frenia, 2007, pp. 107-128.

(13) ABC, viernes 4 de agosto de 1933 , p. 28

(14) Necrología del Ilmo. Sr. D. Ricardo Pérez Valdés por el académico Dr. Va- lle y Aldabalde Anales de la Real Academia Nacional de Medicina (1927); Madrid, Imprenta y encuadernación de Julio Cosano, p. 467-496.

(15) Villasante, O. Las instituciones psiquiátricas madrileñas en el período de entresiglos, Frenia 2005;5:69-99.

(16) Rey, A., José Rodríguez Villargoitia (1811-1854), Rev. Asoc. Esp. Neuropiq 1984;4(10):264-274.

(17) Memoria del Hospital Provincial de Madrid, Madrid, Oficina Tipográfica, 1875, pp. 99-10210.

(18) Real Decreto, Gaceta de Madrid, 21 mayo de 1885, n $^{\circ} 141$, p.511.

(19) González Duro, E., Asistencia psiquiátrica madrileña a fines del siglo XIX, Medicina e historia, 1971, 51, p. 10

(20) López Zanón, A., Historia de un Servicio. Cuadernos de Psiquiatría Madrileña 1971;2(7):12.

(21) Villasante, O. The unfullfilled project of the Model Mental Hospital in Spain: fifty years of the Santa Isabel Madhouse, Leganés (1851-1900). History of Psychiatry 2003;14(1):3-23.

(22) Moro, A Villasante, O. La etapa de Luis Simarro en el Manicomio de Leganés. Frenia 2001;1(1):97-119, p.104.

(23) Huertas, R., Los médicos de la mente. De la Neurología al psicoanálisis: Lafora, Vallejo-Nágera, Garma, Madrid: Nivola, 2002.

(24) Huertas, R., Del Cura, I. De las ciencias básicas a la clínica neuropsiquiátrica: una aproximación a la consulta privada del doctor Lafora, En Martínez Pérez, José; Estévez, Juan; Cura, Mercedes del; Blas Luis V. (eds.). La gestión de la locura: conocimiento, prácticas y escenarios (España, siglos XIX-XX). Cuenca: Universidad Castilla-La Mancha, 2008, pp.151-164.

(25) Moya, G. Gonzalo Rodríguez Lafora. Medicina y cultura en una España en crisis. Madrid: UAM, 1986. 
(26) Villasante, O., Rey, A., Martí, J. V., José $\mathrm{M}^{\mathrm{a}}$ Villaverde: retrato de un desconocido, Medicina e Historia 2008, 1.

(27) Álvarez, R. Higiene mental y eugenesia: La Higiene mental popular de Tomás Busquet. Frenia 2003;3(1):115-147.

(28) Comelles, J. M. La razón y la sinrazón. Barcelona: PPU, 1988.

(29) Martínez Azumendi, O. De visita al Manicomio. Rev. Asoc. Esp. Neuropiq. 2011;31(111):555-561.
(30) Sesión del día 5 de marzo de 1921. Anales de la Real Academia Nacional de Medicina (1921); Madrid, Imprenta y encuadernación de Julio Cosano, p. 109-135. (31) Martín Arnoriaga, T. El terrible caso del psiquiátrico de Valencia. Sábado Gráfico, 18 nov. 1972; 807:31-35. 


\section{ESPAÑA} $\equiv 1916=$

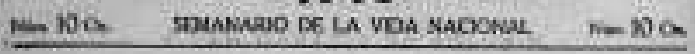

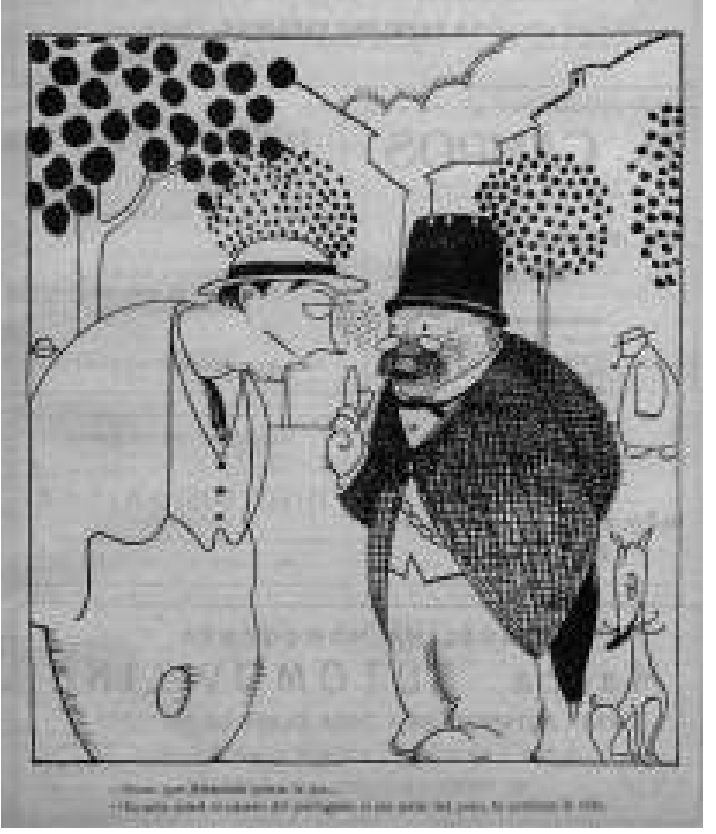

\section{ESPAÑA}

ming

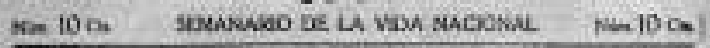

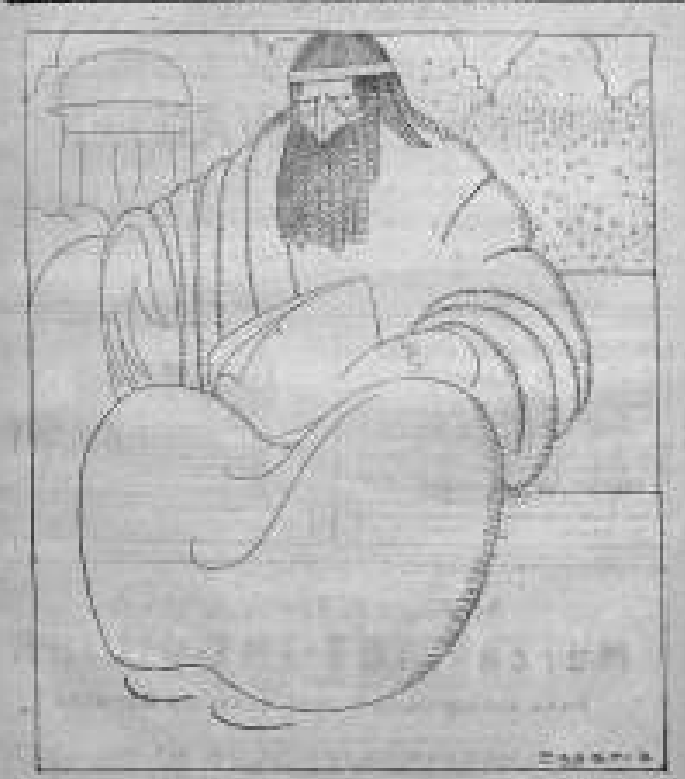

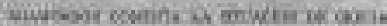

\section{[6. 1400 Res 6... LA CORRESPONDENCIA DE ESPANA}

Siguen los combates en la región del Somme

Ln aumpatis en of Premte de Maeedenia

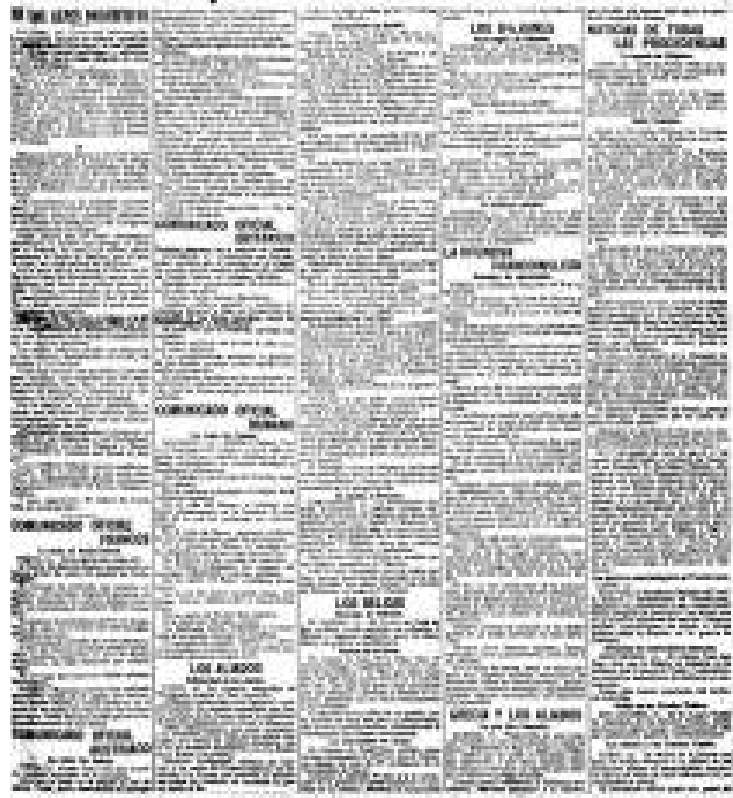

\section{ESPAÑA}

400.

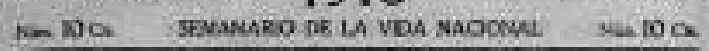

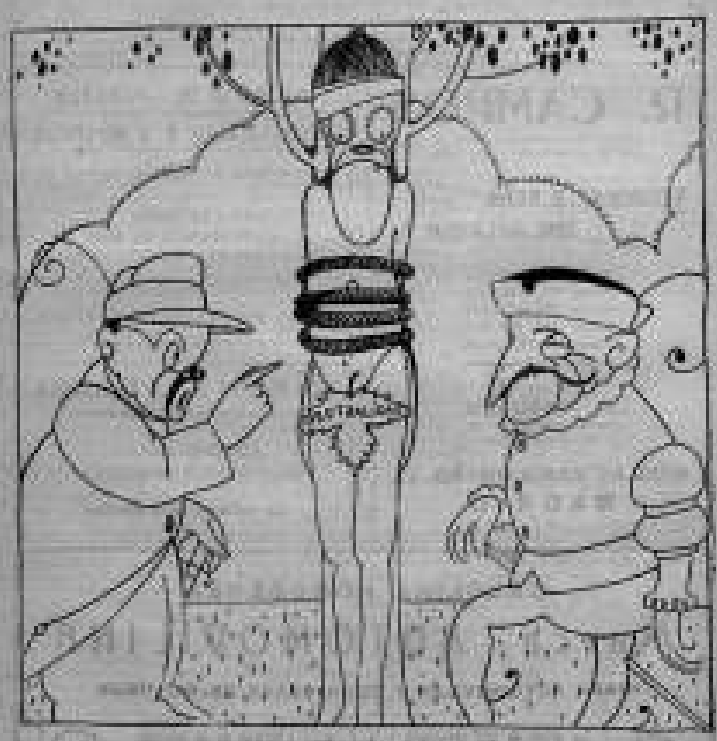

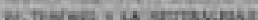

